\title{
Macrophage Accumulation in Atherosclerosis
}

\author{
Brian W. Parks, Ph.D. and Aldons J. Lusis, Ph.D. \\ Departments of Medicine, Human Genetics, and Microbiology, David Geffen School of Medicine, \\ University of California, Los Angeles.
}

\begin{abstract}
Atherosclerosis is a disease of the large arteries that is the major cause of heart disease. It is characterized by the accumulation of cholesteryl esters, macrophages, and fibrous elements in the intimal region underlying the endothelial-cell monolayer. The rupture of such lesions can result in myocardial infarction and the formation of thrombi.
\end{abstract}

Studies in animal models, particularly in mice, have suggested a series of events involved in the development of atherosclerosis. First, plasma lipoproteins accumulate in the intimal region, where they undergo aggregation and modification. This is followed by the entry of monocytes into the intimal region, where they differentiate into macrophages and proceed to take up cholesterol to form cholesterol-engorged foam cells. It has been widely assumed that the rate-limiting event in the accumulation of foam cells during the progression of the disease is the entry of monocytes. However, a recent study by Robbins et al. ${ }^{1}$ shows that the accumulation of macrophage foam cells in established lesions results primarily from macrophage proliferation within the lesion.

The key experiments involved parabiosis, a technique by which two living mice are joined together surgically in such a way that they share a circulatory system. For these experiments, the authors used two different strains of mice: one carrying the cell-surface marker CD45.1 and the other carrying the cell-surface marker CD45.2. The markers are expressed on the surface of monocytes and macrophages, which allowed the investigators to quantitate the number of monocytes and macrophages originating from each strain.

In one experiment, the authors joined hyperlipidemic mice of the two strains, each with established lesions, by means of parabiosis. The mixed blood of the joined mice was chimeric for monocytes and contained substantial levels of both CD45.1-positive and CD45.2-positive cells. After the mice had been joined for 5 weeks, lesional macrophages were observed to exhibit largely the marker type of the host mouse. If monocyte entry into lesions was responsible for macrophage maintenance or expansion, the level of chimerism of the lesional macrophages would have been expected to approximate that of monocytes, but that clearly was not the case (Fig. 1). The authors complemented this experiment with several others that also indicated that in established lesions the replenishment of macrophages depends largely on macrophage proliferation.

The authors also attempted to identify the factors promoting macrophage proliferation within lesions. They found some evidence for a role of scavenger receptor A, although they did not test macrophage colony-stimulating factor, a powerful regulator of macrophage differentiation and proliferation. Macrophage colony-stimulating factor is known to stimulate macrophage proliferation and is produced abundantly in atherosclerotic lesions; its deficiency in mice suppresses the progression of atherosclerotic lesions. ${ }^{2,3}$ Other cell types

Copyright @ 2013 Massachusetts Medical Society.

Disclosure forms provided by the authors are available with the full text of this article at NEJM.org. 
within the lesion, such as T cells, may also contribute to the local proliferation of macrophages. ${ }^{4}$ Clearly, further study is required in order to understand the signals and factors that regulate macrophage proliferation within the atherosclerotic lesion, ${ }^{5}$ and an understanding of how macrophage proliferation contributes to complications of advanced atherosclerosis, such as heart attack and stroke, will be important in the development of new therapeutic agents.

\section{References}

1. Robbins CS, Hilgendorf I, Weber GF, et al. Local proliferation dominates lesional macrophage accumulation in atherosclerosis. Nat Med. 2013; 19:1166-1172.

2. Chitu V, Stanley ER. Colony-stimulating factor-1 in immunity and inflammation. Curr Opin Immunol. 2006; 18:39-48.

3. Smith JD, Trogan E, Ginsberg M, Grigaux C, Tian J, Miyata M. Decreased atherosclerosis in mice deficient in both macrophage colony-stimulating factor (op) and apolipoprotein E. Proc Natl Acad Sci U S A. 1995; 92:8264-8268.

4. Hansson GK, Hermansson A. The immune system in atherosclerosis. Nat Immunol. 2011; 12:204212.

5. Randolph GJ. Proliferating macrophages prevail in atherosclerosis. Nat Med. 2013; 19:1094-1095. 


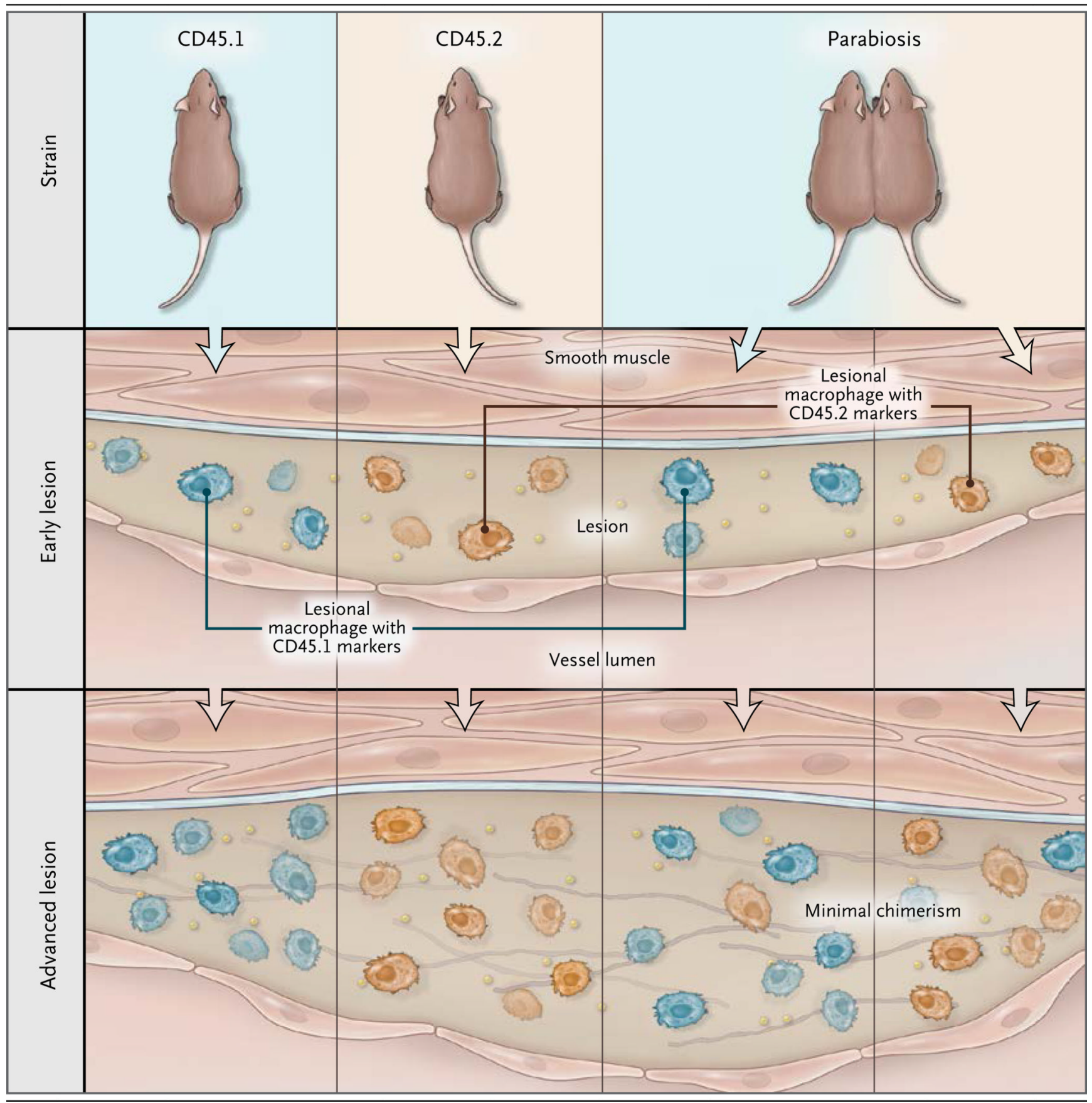

Figure 1. Proliferating Macrophages in Atherosclerosis

Robbins et al. ${ }^{1}$ used parabiosis, the tethering of the circulatory systems of two mice (one in which the monocytes expressed the cell-surface marker CD45.1, and the other in which the monocytes expressed the cell-surface marker CD45.2), to test whether lesional macrophages mirror the chimerism of monocytes present in the combined circulatory system or whether they originate from the host mouse. Robbins et al. found that the lesional macrophages originated predominantly from the host mouse, and they concluded that the proliferation of macrophages within the atherosclerotic lesion is a driver of lesion progression. 